\title{
Childhood Testicular Choriocarcinoma
}

National Cancer Institute

\section{Source}

National Cancer Institute. Childhood Testicular Choriocarcinoma. NCI Thesaurus. Code C6544.

A choriocarcinoma that arises from the testis during childhood. 